\title{
Liderazgo Transformacional y Responsabilidad Social en Asociaciones de Mujeres Cafeteras en el Sur de Colombia
}

\author{
Yenny C. Díaz ${ }^{(1)}$, Juan M. Andrade ${ }^{(1)}$ y Elías Ramírez ${ }^{(2)}$ \\ (1) Corporación Universitaria Minuto de Dios, Neiva, Colombia (e-mail: Ydiazcardoz@uniminuto.edu.co; \\ Jandradenav@uniminuto.edu.co) \\ (2) Universidad Surcolombiana, Neiva, Colombia (e-mail: Elias.ramirez@usco.edu.co)
}

Recibido Nov. 23, 2018; Aceptado Ene. 21, 2019; Versión final Feb. 24, 2019, Publicado Oct. 2019

\begin{abstract}
Resumen
El presente estudio estableció la relación que existe entre el liderazgo transformacional y las prácticas de responsabilidad social en organizaciones de mujeres productoras de cafés especiales en el sur de Colombia. Al respecto, se aplicaron dos cuestionarios para medir las variables mediante escalas Likert y se utilizó el Modelo de Ecuaciones Estructurales para validar la hipótesis con el software SPSS - AMOS. Se determinó una relación significativa y positiva entre las prácticas de liderazgo transformacional y la percepción de responsabilidad social, especialmente en las dimensiones de influencia idealizada (conducta y atribuida) y estimulación intelectual del liderazgo transformacional, y las dimensiones de asuntos laborales y sociedad de la responsabilidad social. Estos hallazgos son congruentes con la literatura actual, y complementa aspectos no abordados como la relación entre las variables estudiadas en el marco del género en organizaciones no convencionales.
\end{abstract}

Palabras clave: asociaciones cafeteras; mujeres; liderazgo transformacional; responsabilidad social.

\section{Transformational Leadership and Social Responsibility in Coffee Women's Associations in Southern Colombia}

\begin{abstract}
The present study established the relationship between transformational leadership and social responsibility practices in organizations of women, producers of specialty coffees in southern Colombia. Two questionnaires were applied to measure the variables using Likert scales and the structural equations model was used to validate the hypothesis with SPSS-AMOS software. A significant and positive relationship was determined between transformational leadership practices and the perception of social responsibility, especially in the dimensions of idealized influence (conduct and attribution) and intellectual stimulation of transformational leadership, and the dimensions of labor issues and social responsibility society. These findings are consistent with current literature, and they complement aspects not addressed as the relationship between the variables studied in the framework of gender in non-conventional organizations.
\end{abstract}

Keywords: coffee associations; women; transformational leadership; social responsibility 


\section{INTRODUCCIÓN}

Pese a ser un tema ampliamente estudiado, el liderazgo transformacional ha mostrado ser uno de los planteamientos con mayor relevancia e impacto a nivel investigativo (Judge y Piccolo, 2004) y gerencial (Bono y Judge, 2004), recibiendo gran atención durante las últimas tres décadas, y constituyéndose como una de las teorías más dominantes del liderazgo (Mhatre y Riggio, 2014). Esto, principalmente, por la relación que la investigación ha demostrado entre dicho estilo de liderazgo y las actitudes y comportamientos de los colaboradores, en particular, y la organización, en general (Cóndor et al., 2018). Recientes estudios, por ejemplo, relacionan el estilo de liderazgo transformacional de los gerentes con resultados y comportamientos positivos de los empleados en aspectos tales como actitud (Lee et al., 2013), lealtad (Lee, 2005), compromiso organizacional (Cole y Bedeian, 2007), creatividad e innovación (Mohamed, 2016), desempeño (Aga et al., 2016) y responsabilidad social (Luu, 2012), entre otros. Frente a la relación entre liderazgo transformacional y responsabilidad social, son varios los estudios recientes que han buscado confirmarla, y aunque existe cierta evidencia empírica y teórica, se hace preciso continuar fortaleciendo sus hallazgos. Al respecto, entre los aspectos menos abordados en dicha relación, se encuentran el género (Almeida et al., 2017) y las pequeñas organizaciones (Angus-Leppan et al., 2010), por lo que se hace necesario incluir dichos aspectos en su estudio.

Para Bass y Avolio (1994), el liderazgo transformacional se refiere a un conjunto de comportamientos del líder que transforman las necesidades de sus seguidores mediante la autorrealización, estimulando sus expectativas a niveles superiores. La transformación se basa en la premisa de que el liderazgo transformacional fomenta el desarrollo de los empleados e incrementa los niveles de motivación y la moral de los seguidores (Burns, 1978). De acuerdo a sus planteamientos, los líderes transformacionales poseen cuatro atributos o dimensiones de comportamiento: influencia idealizada (que algunos autores llaman carisma), motivación inspiradora, estimulación intelectual y consideración individualizada (Bass, 1985). La influencia idealizada describe a los líderes que tienen gran poder e influencia para ser modelos de conducta para sus seguidores. Los líderes transformacionales tienen altos estándares de conducta moral y ética, y proporcionan una visión y un sentido de misión para los seguidores. Algunos estudios posteriores clasifican la influencia idealizada en atribuida y comportamiento (Avolio y Bass, 2004). La segunda dimensión, motivación inspiradora, implica la estimulación y aumento de la motivación más allá de las expectativas originales. Aquí, el líder articula una visión atractiva para los seguidores y transmite optimismo sobre los objetivos futuros. Por su parte, la estimulación intelectual representa un liderazgo que estimula a los seguidores a pensar los viejos problemas de formas nuevas, proporcionándoles autonomía y empoderamiento en el trabajo; en otras palabras, busca aumentar la conciencia de los seguidores sobre los problemas e influir en ellos para desarrollar enfoques innovadores o creativos en su resolución (Judge y Piccolo, 2004). Finamente, la dimensión consideración individualizada involucra el apoyo y receptividad del líder frente a las necesidades individuales de los seguidores, así como su rol de mentor frente a cada uno (Avolio et al., 2004). Al respecto, algunos estudios han asociado el liderazgo transformacional más con las mujeres, que con los hombres, debido a que enfatiza en las capacidades intelectuales y sociales, y la creatividad, y menos en el deseo de ejercer control, poder y otorgar recompensas, estos últimos aspectos vinculados generalmente a estilos masculinos (Alimo-Metcalfe, 2010).

De otra parte, la responsabilidad social se ha convertido recientemente en un campo de interés (Serenko y Bontis, 2009) y a medida que evoluciona son múltiples los estudios que se han realizado con diferentes fines, algunos con el objetivo de medir sus prácticas (Wood, 2010), su impacto sobre el desempeño financiero de las organizaciones y su operacionalización (Padilla et al., 2017). Otros autores han centrado su atención en establecer sus vínculos con variables organizacional (Aguinis, 2011). A partir del enfoque institucional, las empresas realizan responsabilidad social por presión de grupos interesados (Stevens et al., 2005), que bien pueden ser accionistas (David et al., 2007); consumidores (Christmann y Taylor, 2006); medios de comunicación (Weaver et al., 1999); la comunidad local (Marquis et al., 2007) o grupos de interés (Greening y Gray, 1994). Autores afines al enfoque organizacional consideran que las empresas participan de la responsabilidad social por una motivación instrumental (Bansal y Roth, 2000). Asimismo, aspectos empresariales influyen en las iniciativas responsables como tener una misión y valores afines o una gerencia equitativa y comprometida (Marcus y Anderson, 2006). El enfoque de análisis a nivel individual incluye aspectos ligados a las capacidades de los líderes y gerentes (Sully de Luque et al., 2008) y las necesidades de los colaboradores (Aguilera et al., 2007). Al respecto, existe un interés creciente en realizar estudios de prácticas de responsabilidad social en las pequeñas empresas dada sus características particulares en asuntos sociales y ambientales (Johnson y Schaltegger, 2016), llegando incluso a plantearse propuestas como la Responsabilidad Social de la Pequeña Empresa (SBSR) (Lepoutre y Heene, 2006). Frente a la importancia del género en la responsabilidad social, investigaciones recientes, han evidenciado que organizaciones con juntas directivas o gerentes mujeres desarrollan mayores niveles de percepción y prácticas de responsabilidad social, identificando actitudes asociadas más positivas que entre los hombres, especialmente frente a los colaboradores de la organización (Larrieta et al., 2015). 
La escasa investigación sobre el tema demuestra que el liderazgo transformacional está asociado con un alto nivel de responsabilidad social (Angus-Leppan et al., 2010), en el entendido de que los gerentes, particularmente en posiciones principales, pueden influir en la responsabilidad social de las organizaciones (Waldman y Siegel, 2008). Al respecto, Maak et al. (2016, p. 467) afirman que "las organizaciones pueden entenderse como un reflejo de sus altos ejecutivos" y, por tanto, el enfoque de una organización está influido por el estilo de liderazgo de sus directivos (Almeida et al., 2017). Así, las investigaciones evidenciaron que el liderazgo transformacional está directa o indirectamente vinculado a las percepciones positivas de la RS y se implementa para equilibrar los intereses de la mayoría de las partes interesadas. De igual manera, Waldman et al. (2006) encontraron que el liderazgo visionario está asociado con ciertos valores de gestión que los gerentes a menudo aplican en sus procesos de toma de decisiones relevantes para la RS. De otra parte, Goswami et al. (2018) mostraron evidencia empírica del rol del liderazgo transformacional como mediador entre las percepciones de responsabilidad social y el comportamiento ciudadano organizacional de los colaboradores.

En síntesis, de los enfoques de liderazgo, especialmente los que surgieron a partir la década de 1980, el liderazgo transformacional parece ser de particular importancia para explicar el vínculo entre la integridad y las prácticas de RS, especialmente en sus dimensiones estimulación intelectual e inspiración motivacional (Waddock y Bodwell 2007). A partir de lo expuesto, el presente trabajo busca determinar la relación entre el liderazgo transformacional y la responsabilidad social en las organizaciones de mujeres productoras de cafés diferenciados, teniendo en cuenta que son pocos los estudios que contemplan organizaciones compuestas exclusivamente por mujeres en contextos de países en desarrollo en el ámbito rural. Por lo anterior se plantea la siguiente hipótesis de trabajo: "El liderazgo transformacional se relaciona significativa y positivamente con la responsabilidad social en las asociaciones de mujeres productoras de cafés diferenciados".

\section{METODOLOGÍA}

La metodología se presenta en cuatro subsecciones: en la primera se describe el tipo y corte de la investigación; en la segunda sección se describen los participantes en la investigación y algunas características sociodemográficas; en la tercera se detallan las variables de estudio y sus componentes; y en la cuarta el software para el análisis de la información.

\section{Tipo de investigación}

Se realizó una investigación transversal, correlacional y cuantitativa (Hernández et al., 2010), para estudiar la relación entre el liderazgo transformacional y las prácticas de responsabilidad social desarrolladas en las asociaciones de mujeres productoras de cafés especiales en el sur de Colombia.

\section{Participantes}

Se realizó la investigación en organizaciones de mujeres productoras de cafés especiales en el sur colombiano, en las cuales se aplicaron 450 encuestas a sus asociadas en 16 organizaciones legalmente constituidas. La muestra estuvo compuesta exclusivamente por mujeres, el rango de edad osciló entre los 26 y 80 años. Con respecto al nivel de educación, sobresale un bajo nivel académico donde el $24 \%$ no tiene educación formal, el $52 \%$ acredita primaria, el 18\% tiene secundaria, el 4,0\% alcanzó un nivel de formación técnica y el $2 \%$ formación profesional. El $48 \%$ de las mujeres son cabeza de hogar y el $92 \%$ tienen entre 1 y 12 personas a cargo. La fuente del ingreso económico familiar se compone exclusivamente del cultivo de café (100\%). Los estratos que predominan son el 1 con el $68.5 \%$ y 2 con el $31 \%$. El 59,4\% tienen predios con extensión entre 1 y 5 hectáreas, y el $53 \%$ son propietarias.

Frente a las certificaciones adquiridas, el porcentaje más representativo es la certificación Fair Trade - FLO (37\%) que fomenta el comercio justo para pequeños productores y el acceso a mercados equitativos. En cuanto a los códigos de conducta, las asociaciones poseen mayor orientación a 4C, que estipula criterios aplicables a la producción, elaboración y comercialización sostenible de café verde (20\%). En un menor porcentaje se identifican UTZ Certified (13\%), Rainforest Allience (10\%), C.A.F.E practices (3\%), Certified Organic (3\%) y Nespresso AAA (0,6\%).

Con respecto a las consideraciones éticas, el estudio protegió la privacidad de las personas sujeto de investigación puesto que en ningún momento se identificaron, por lo tanto, permanecieron en el anonimato y por ninguna razón ni medio fueron divulgados sus nombres. Asimismo, ningún procedimiento se inició sin el previo consentimiento voluntario. En efecto, en el consentimiento informado se describió la naturaleza de los procedimientos, la importancia y la finalidad del estudio sobre la confidencialidad de la información y sus posibles usos. 


\section{Medidas}

Para medir el liderazgo transformacional se utilizó el Multifactor Leadership Questionnaire 5X versión corta (MLQ5X) de Bass y Avolio (1995). EI MLQ-5X es uno de los instrumentos de medición del liderazgo más utilizado a nivel mundial y ha sido empleado por diversas investigaciones en contextos de habla hispana en general (p.ej. Cuadrado y Molero, 2002) y en Colombia en particular. Esta versión contiene treinta y dos ítems para medir el liderazgo tanto transformacional como transaccional. Para los todos los ítems, las personas indicaron su percepción en una escala tipo Likert de cinco puntos, donde uno (1) significa totalmente en desacuerdo y cinco (5) significa totalmente de acuerdo. Por otro lado, la medición de la responsabilidad social se realizó con un instrumento elaborado por los investigadores con fundamento en el proceso de auscultación teórica, revisión y análisis de estándares asociados, sometida a proceso de validez del constructo a través de grupos focales, validación con expertos y definición operativa de las variables. El cuestionario cuenta con 29 ítems derivados de 4 dimensiones: valores, transparencia y gobierno, asuntos laborales, medio ambiente, y sociedad. Para los todos los ítems, las personas indicaron su percepción en una escala tipo Likert de cinco puntos, donde uno (1) significa totalmente en desacuerdo y cinco (5) significa totalmente de acuerdo (ver tabla 1).

Tabla 1: Definición y operación de variables de los cuestionarios

\begin{tabular}{|c|c|c|c|}
\hline Variable & \multicolumn{2}{|r|}{ Definición } & Definición \\
\hline \multirow{5}{*}{$\begin{array}{l}\text { Liderazgo } \\
\text { Transformacion } \\
\text { al } \\
\text { (LTF) }\end{array}$} & \multirow{2}{*}{ 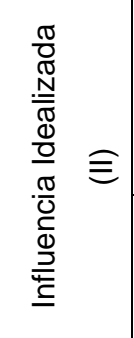 } & $\begin{array}{c}\text { Influencia } \\
\text { Idealizada } \\
\text { (Atribuida) } \\
\text { (II-A) }\end{array}$ & $\begin{array}{l}\text { El líder obtiene la confianza de los seguidores. Estos se sienten } \\
\text { identificados y tienden a imitarlos. }\end{array}$ \\
\hline & & $\begin{array}{l}\text { Influencia } \\
\text { Idealizada } \\
\text { (Conducta) }(\mathrm{II-C})\end{array}$ & Difiere del anterior, al centrarse a conductas específicas. \\
\hline & \multicolumn{2}{|c|}{ Inspiración motivacional (IM) } & $\begin{array}{l}\text { Capacidad de motivar a los demás para alcanzar un } \\
\text { desempeño superior. Crea una visión estimulante y atractiva } \\
\text { para sus seguidores. Busca esfuerzos extras y alienta al } \\
\text { cambio. }\end{array}$ \\
\hline & \multicolumn{2}{|c|}{ Estimulación intelectual (EI) } & $\begin{array}{l}\text { Permite empoderar a otros para que piensen sobre los } \\
\text { problemas y desarrollen habilidades para resolverlos. }\end{array}$ \\
\hline & \multicolumn{2}{|c|}{ Consideración individual (Cl) } & $\begin{array}{l}\text { Es un escucha activo y comunicador fuerte. Proporciona } \\
\text { cuidado y empatía, y provee retos y oportunidades para los } \\
\text { otros. }\end{array}$ \\
\hline \multirow{4}{*}{$\begin{array}{l}\text { Responsabilidad } \\
\text { Social } \\
\text { (RS) }\end{array}$} & \multicolumn{2}{|c|}{$\begin{array}{l}\text { Valores, transparencia y } \\
\text { gobierno } \\
\text { (V) }\end{array}$} & $\begin{array}{l}\text { Refiere la adopción y alcance de valores y principios éticos, } \\
\text { prácticas de gobierno y autorregulación de la conducta, como } \\
\text { relaciones transparentes con la sociedad. }\end{array}$ \\
\hline & \multicolumn{2}{|c|}{$\begin{array}{l}\text { Asuntos laborales } \\
\qquad(\mathrm{AL})\end{array}$} & $\begin{array}{l}\text { Políticas y prácticas internas orientadas al trabajo o la } \\
\text { asociatividad. }\end{array}$ \\
\hline & \multicolumn{2}{|c|}{$\begin{array}{l}\text { Medio ambiente } \\
\qquad(\mathrm{MA})\end{array}$} & $\begin{array}{l}\text { Adopción de un enfoque integrado orientado a la mitigación de } \\
\text { las implicaciones directas e indirectas al medio ambiente. }\end{array}$ \\
\hline & \multicolumn{2}{|r|}{$\begin{array}{l}\text { Sociedad } \\
\text { (S) }\end{array}$} & $\begin{array}{l}\text { Acciones que promuevan el relacionamiento con las partes } \\
\text { interesadas (sociedad), el liderazgo, los derechos humanos }\end{array}$ \\
\hline
\end{tabular}

\section{Análisis de datos}

En términos generales, para detectar los efectos propuestos en la hipótesis de investigación y evaluar las relaciones y efectos entre las variables se utilizó un Modelo de Ecuaciones Estructurales (Structural Equation Modeling o SEM). EI SEM es una técnica estadística de análisis multivariado que permite poner a prueba relaciones causales-usando datos cuantitativos-fundamentadas en supuestos o reflexiones cualitativas sobre el sentido de la causalidad (Hair et al., 1999). 


\section{RESULTADOS}

A continuación, se presenta el análisis factorial confirmatorio sobre los datos actuales. Se empleó el método de estimación de máxima verosimilitud y el análisis se realizó utilizando una matriz de covarianza. El modelo teórico comprendía el liderazgo transformacional y las prácticas de responsabilidad social percibida como variables latentes y sus respectivos indicadores. Al respecto, el modelo obtuvo un buen ajuste a los datos, obteniendo un chi-cuadrado significativo $\left(\mathrm{X}^{2}[1118, \mathrm{~N}=450]=2456,438 ; \mathrm{p}<0,00\right), \mathrm{IFI}=.91, \mathrm{TLI}=.90, \mathrm{CFI}=$ $0.91, \mathrm{GFI}=0.89$, lo que denota que el modelo propuesto se acerca mucho al modelo obtenido. De otra parte, en la tabla 2 se aprecian en paréntesis el Alfa de Cronbach para las dos variables propuestas, evidenciando una excelente consistencia (Liderazgo transformacional, $\alpha=0.95$; Responsabilidad social, $\alpha=0,90$ ).

Tabla 2: Medias, desviaciones estándar y correlaciones de las variables de estudio

\begin{tabular}{ccccc}
\hline Variable & $M$ & $D E$ & 1 & 2 \\
\hline 1. Liderazgo Transformacional - LTF & 3,84 & 0,68 & $(0,95)$ & $(0,90)$ \\
2. Responsabilidad Social - RS & 3,28 & 0,91 & $0,739^{* *}$ &
\end{tabular}

Además, se realizó un análisis más detallado de la confiabilidad de las dimensiones del instrumento (ver tabla 3). Para las dimensiones del liderazgo transformación fue: influencia idealizada (atribuida y conducta) con 8 elementos fue de 0,97 , inspiración motivacional con 4 elementos fue de 0,94, consideración individualizada con 4 elementos fue de 0,92, y estimulación intelectual con 4 elementos fue de 0,93 , mientras para las dimensiones de la responsabilidad social fue: medio ambiente con 9 elementos fue de 0,90 , sociedad con 7 elementos fue de 0,88 , valores, transparencia y gobierno con 7 elementos fue de 0,89 , y asuntos laborales con 6 elementos fue de 0,92. Como se observa, en todos los casos los coeficientes representan un índice adecuado de convergencia. Posteriormente, se muestran las correlaciones del estudio a través del modelo de ecuación estructural. Todas las correlaciones fueron en la dirección esperada y proporcionaron apoyo preliminar para nuestra hipótesis.

Tabla 3: Confiabilidad de las dimensiones del cuestionario

\begin{tabular}{|lcc|}
\hline Estadísticas de fiabilidad & Alfa de Cronbach & N elementos \\
\hline Influencia Idealizada - II & $\alpha=0,97$ & 8 \\
Inspiración motivacional - IM & $\alpha=0,94$ & 4 \\
Consideración individual - CI & $\alpha=0,92$ & 4 \\
Estimulación Intelectual - EI & $\alpha=0,93$ & 4 \\
Medio Ambiente - MA & $\alpha=0,90$ & 9 \\
Sociedad - S & $\alpha=0,88$ & 7 \\
Valores, transparencia y gobierno -V & $\alpha=0,89$ & 7 \\
Asuntos Laborales - AL & $\alpha=0,92$ & 6 \\
\hline
\end{tabular}

Como se puede observar en la Figura 1 , existe una relación positiva y significativa entre liderazgo transformacional y la responsabilidad social: 0,$739 ; p<0,00$. De lo que se puede inferir que entre más aumente el nivel de liderazgo transformacional, mayor será la percepción de responsabilidad social de las seguidoras en la organización. Asimismo, las correlaciones del liderazgo transformacional son significativas, especialmente la influencia idealizada $(0,92 ; p<0,00)$ y estimulación intelectual $(0,97 ; p<0,00)$, y en menor medida la inspiración motivadora $(0,91 ; p<0,00)$ y consideración individualizada $(0,73 ; p<0,00)$, mientras en las dimensiones de la responsabilidad social las mayores correlaciones se presentaron en los asuntos laborales $(0,99 ; p<0,00)$ y sociedad $(0,92 ; p<0,00)$, seguidas de valores $(0,81 ; p<0,00)$ y medio ambiente $(0,72 ; p<0,00)$ (ver tabla 4).

Con relación a la tabla 4 y la figura 1, se debe advertir que las relaciones se hipotetizaron entre las variables denominadas liderazgo transformacional y responsabilidad social, no entre sus componentes o dimensiones, por lo anterior la investigación, si bien tuvo en cuenta sus dimensiones, no estableció relaciones entre estas, al menos no como hipótesis de trabajo. Ahora, las dimensiones se relacionaron con su respectiva variable, porque se modeló la hipótesis y es importante determinar la fuerza de la relación entre las dimensiones del liderazgo transformacional al momento de vincularse con la responsabilidad social y viceversa. Al respecto, se deseaba establecer su comportamiento cuando se establecieron relaciones entre variables de manera simultánea. 


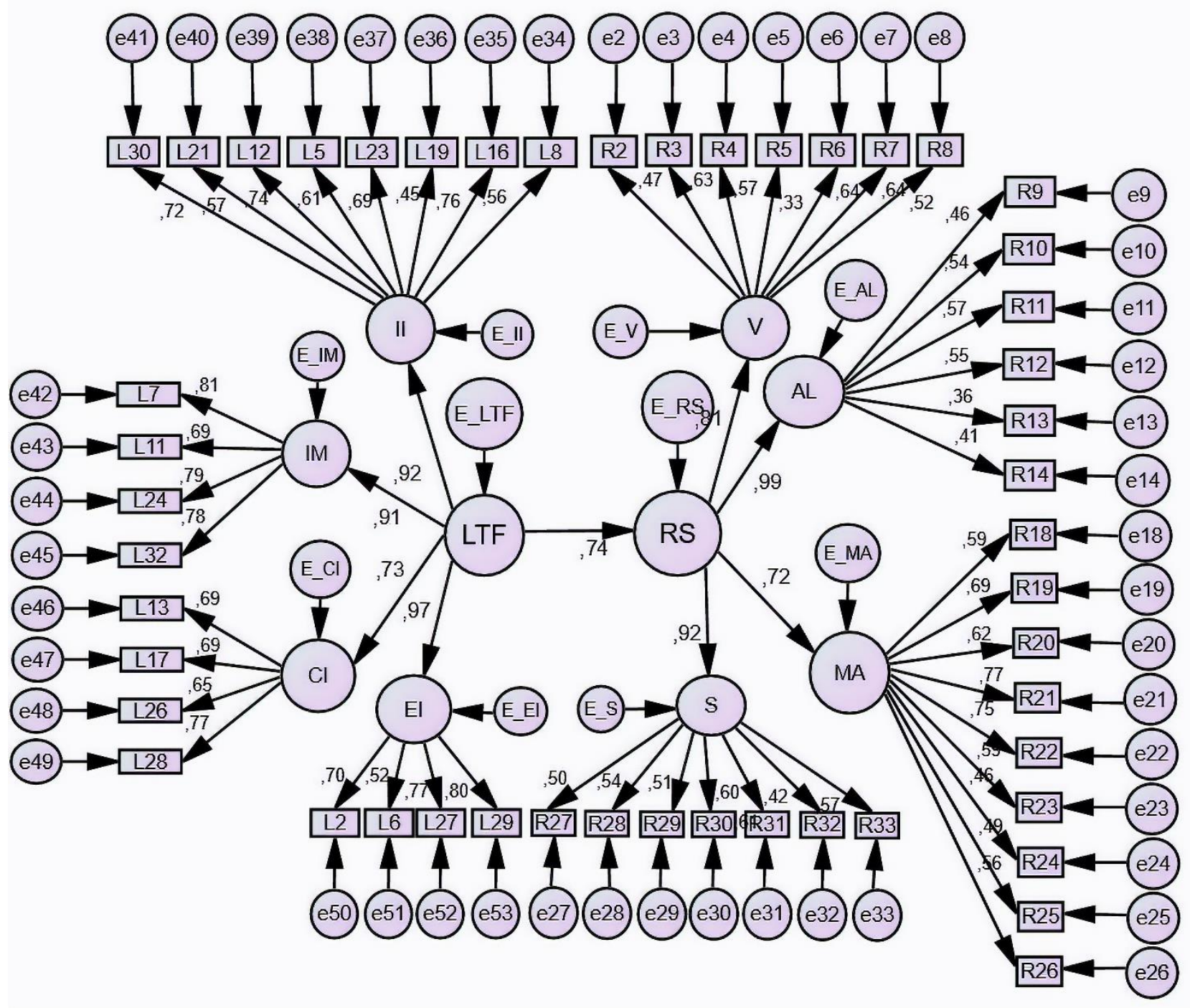

Fig. 1: Resultados del modelo de ecuaciones estructurales

Tabla 4: Correlaciones entre las variables y sus dimensiones

\begin{tabular}{|c|c|c|c|}
\hline \multicolumn{3}{|c|}{ Relación } & Estimación \\
\hline Responsabilidad social - RS & $\begin{array}{ll}<---- \\
\end{array}$ & Liderazgo Transformacional - LTF &, $739^{* * *}$ \\
\hline Influencia Idealizada - II & $<----$ & Liderazgo Transformacional - LTF &, $921^{* \star *}$ \\
\hline Inspiración motivacional - IM & $<----$ & Liderazgo Transformacional - LTF &, $912^{\star \star *}$ \\
\hline Consideración individual - Cl & $<---$ & Liderazgo Transformacional - LTF &, $731^{* * *}$ \\
\hline Estimulación Intelectual - El & $<---$ & Liderazgo Transformacional - LTF &, $970 * * *$ \\
\hline Medio Ambiente - MA & $<---$ & Responsabilidad social - RS &, $723^{\star \star *}$ \\
\hline Sociedad - S & $<---$ & Responsabilidad social - RS &, $917^{\star \star \star}$ \\
\hline Valores, transparencia y gobierno -V & $<---$ & Responsabilidad social - RS &, $812^{* * *}$ \\
\hline Asuntos Laborales - AL & $<----$ & Responsabilidad social - RS &, $991^{* * *}$ \\
\hline
\end{tabular}

\section{DISCUSIÓN}

Frente a las variables de liderazgo transformacional y responsabilidad social, existe una relación positiva y significativa, lo que indica que, a mayor percepción de liderazgo transformacional, mayor será la percepción de acciones de responsabilidad social dentro de las asociaciones. Inicialmente, se puede deducir que las líderes transformacionales han alcanzado altos niveles de desarrollo moral, que se ven reflejados en el análisis descriptivo significativo de la dimensión valores, transparencia y gobierno (responsabilidad social), que articula una visión justa y sincronizada de las necesidades de los grupos de interés; haciendo que el liderazgo trascienda del propio interés a una visión más amplia y competitiva desde un modelo de responsabilidad social. Por tanto, la relación significativa entre la influencia idealizada atribuida y la dimensión valores, transparencia y gobierno, permite deducir que entre más confianza genera la líder a sus seguidoras, 
y mientras las seguidoras se sientan identificadas con ella, más se consideran la adopción y alcance de prácticas de gobierno y autorregulación de la conducta, como relaciones transparentes con la sociedad, lo que evidencia la alta correlación $(0,92 ; p<0,00)$ de la dimensión sociedad.

De otra parte, la búsqueda de interiorización de los valores y el comportamiento ético a sus seguidoras se ve reflejado en la influencia idealizada promulgada por la líder, generando una imagen de confianza, que permite que se sientan identificadas y tiendan a imitarlas, tal como lo sostiene Maak et al. (2016); no obstante, estén más centradas en las conductas específicas, que una influencia atribuida. Sin embargo, para el caso estudiado la dimensión valores no estuvo dentro de los mayores índices de correlación, aunque con una correlación altamente significativa $(0,81 ; p<0,00)$. Al respecto, en concordancia con lo expresado por Waddock y Bodwell (2007), se puede establecer una marcada relación entre todas las dimensiones de responsabilidad social con la estimulación intelectual. Lo anterior se explica desde el rol de las líderes incitando a las asociadas a la solución de problemas de manera creativa, especialmente en situaciones como la confrontación de dinámicas familiares y sociales tradicionales, las prácticas puntuales de cultivo y postcosecha, entre otras, que les ha permitido desarrollar habilidades en el marco prácticas socialmente responsables para la solución de estas. Por lo cual se puede explicar el alto nivel de correlación de la dimensión estimulación intelectual $(0,97 ; p<0,00)$.

Por otro lado, la fuerte correlación de la dimensión de asuntos laborales se puede explicar especialmente en la naturaleza y misión de la organización, dado que se mueve en el mercado de los cafés especiales y diferenciados, donde las certificaciones de comercio justo y buenas prácticas determinan el éxito del negocio. Así, a la luz de lo propuesto por Christmann y Taylor (2006), las empresas compradoras del café especial generan presión para que la gerencia y las líderes de las organizaciones establezcan retribuciones económicas justas con las asociadas y prácticas cordiales con las comunidades aledañas. En ese mismo sentido, las líderes deben orientar su gestión a afianzar las prácticas laborales como retribuciones diferenciadas, bienestar de las asociadas, capacitaciones y formación, entre otros, lo que de alguna manera define la permanencia de las asociadas, tal como lo sostienen David et al. (2007) al considerar que la presión de los interesados, en este caso las asociadas, exige unas prácticas laborales benéficas para ellas.

En efecto, las líderes tienden a buscar satisfacer metas organizacionales y personales. Esto, frente a la responsabilidad social, permite ver el grado de significancia que se le otorga a través de las cuatro prácticas de responsabilidad social mencionadas. Además, se establece un sentimiento de reciprocidad entre las líderes y seguidoras, generando consideraciones individuales ante el cuidado, la empatía, la provisión de retos y oportunidades para cada una, que se trazan en las acciones para el fortalecimiento de la asociación. Llama la atención que la dimensión medio ambiente tuvo la correlación más baja con respecto a la responsabilidad social; no obstante, fue significativa $(0,72 ; p<0,00)$, situación que contrasta con uno de los planteamientos más fuertes de la responsabilidad social que es su énfasis en las condiciones ambientales.

Finalmente, frente a las posibles diferencias que puedan existir entre los resultados obtenidos en esta investigación y los obtenidos en estudios realizados con organizaciones dominadas por hombres, no se advierten diferencias significativas, en concordancia con lo hallado por Almeida et al. (2017), aunque se debe profundizar las investigaciones al respecto, auscultando otros tipos de liderazgo.

\section{CONCLUSIONES}

1. En general se confirma una relación positiva y significativa entre la variable liderazgo transformacional y las prácticas de responsabilidad social percibida en las organizaciones de mujeres productoras de cafés especiales en el sur de Colombia.

2. Las dimensiones que tienen mayor correlación con el liderazgo transformacional son la influencia idealizada (conducta y atribuida) y estimulación intelectual, lo que se puede explicar desde la capacidad de las líderes de ser ejemplo para su seguidora, para el primer caso, y desde el estímulo para que las seguidoras ausculten soluciones a los problemas acaecidos en la cotidianidad del cultivo de café como la siembra, riego, recolección, entre otros, en el segundo. De igual manera, las dimensiones de mayor correlación con la variable responsabilidad social fueron asuntos laborales y sociedad, denotando la importancia de las condiciones de trabajo y la remuneración, y el vínculo con la comunidad que tiene la organización.

3. Se torna interesante que se profundice en la influencia de otras modalidades de liderazgo que no fueron considerados en este estudio y que la teoría considera que son significativos. Igualmente podría explorarse el efecto conjunto de otras variables claramente asociadas al estilo de liderazgo transformacional como la confianza, el soporte organizacional, la justicia y el compromiso, en el marco de organizaciones no convencionales.

4. Con relación a las limitaciones del estudio, una primera se relaciona con que los datos fueron obtenidos mediante una investigación transversal; es decir, que los datos fueron recogidos en un momento específico 
del tiempo y no se contempló la evolución o relación gradual de fenómeno a lo largo del tiempo. Por lo cual, se necesitarían estudios longitudinales para tratar auscultar el comportamiento de los resultados. Otra limitación resulta en que la investigación contempló el análisis de un solo estilo de liderazgo; esto es, el liderazgo transformacional, cuando la literatura de liderazgo ha comenzado a plantear y analizar el efecto de otros estilos de liderazgo tales como el liderazgo ético, el liderazgo de servicio, el liderazgo auténtico y el liderazgo responsable

\section{REFERENCIAS}

Aga, D.A., N. Noorderhaven y B. Vallejo, Transformational leadership and project success: The mediating role of teambuilding, doi: 10.1016/j.jproman.2016.02.012, International Journal of Project Management, 34, 806-818 (2016)

Aguilera, R.V., D.E. Rupp, C.A. Williams y J. Ganapathi, Putting the $S$ back in corporate social responsibility: A multilevel theory of social change in organizations, doi: 10.2307/20159338, Academy of Management Review, 32, 836-863 (2007)

Aguinis, H., Organizational responsibility: Doing good and doing well; In S. Zedeck (Ed.), APA handbook of industrial and organizational psychology, 3, 855-879, American Psychological Association, Washington, DC, USA (2011)

Alimo-Metcalfe, B., An investigation of female and male constructs of leadership and empowerment, doi: 10.1108/17542411011092309, Gender in Management: An International Journal, 25(8), 640-648 (2010)

Almeida, M.M., J. Perramon y L. Bagur-Femenias, Leadership styles and corporate social responsibility management: Analysis from a gender perspective, doi: 10.1111/beer.12060, Business Ethics a European Review, 1-15 (2017)

Angus-Leppan, T., L. Metcalf y S. Benn, Leadership styles and CSR practice: An examination of sensemaking, institutional drivers and CSR leadership, doi: 10.1007/s10551-009-0221-y, Journal of Business Ethics, 93(2), 189-213 (2010)

Avolio, B.J. y B.M. Bass, Multifactor Leadership Questionnaire, 3rd Ed., Mind Garden, Palo Alto, USA (2004)

Avolio, B.J., W. Zhu, W. Koh y P. Bhatia, Transformational leadership and organizational commitment: mediating role of psychological empowerment and moderating role of structural distance, doi: 10.1002/job.283, Journal of Organizational Behavior, 25(8), 951-968 (2004)

Bansal, P. y K. Roth, Why companies go green: A model of ecological responsiveness, doi: 10.2307/1556363, Academy of Management Journal, 43, 717-736 (2000)

Bass, B.M. y B.J. Avolio, (Eds.), Improving organizational effectiveness through transformational leadership, Sage Publications, Thousand Oaks, USA (1994)

Bass, B.M. y B.J. Avolio, Multifactor Leadership Questionnaire, Mind Garden, Palo Alto, USA (1995)

Bass, B.M., Comment: Transformational leadership. Looking at other possible antecedents and consequences, doi: 10.1177/105649269543010, Journal of Management Inquiry, 4, 293-297 (1985)

Bono, J.E. y T.A. Judge, Personality and Transformational and Transactional Leadership: A Meta-Analysis, doi: 10.1037/0021-9010.89.5.901, Journal of Applied Psychology, 89(5), 901-910 (2004)

Christmann, P. y G. Taylor, Firm self-regulation through international certifiable standards: Determinants of symbolic versus substantive implementation, doi: 10.1057/palgrave.jibs.8400231, Journal of International Business Studies, 37, 863-878 (2006)

Cole, M.S. y A.G. Bedeian, Leadership consensus as a cross-level contextual moderator of the emotional exhaustion-work commitment relationship, doi: 10.1016/j.leaqua.2007.07.002, The Leadership Quarterly, 18, 447-462 (2007)

Cóndor, E.G., M.A. Bustamante, M.C. Lapo y R.M. Campos, Factores Intrínsecos de Motivación y Liderazgo en una Empresa Familiar Ecuatoriana de Telecomunicaciones, Revista Información Tecnológica, 29(4), 205-216 (2018)

David, P., M. Bloom y A.J. Hillman, Investor activism, managerial responsiveness, and corporate social performance, doi: 10.1002/smj.571, Strategic Management Journal, 28, 91-100 (2007)

Goswami, A., K.E. O'Brien, K. Dawson y M. Hardiman, Mechanisms of Corporate Social Responsibility: The Moderating Role of Transformational Leadership, doi: 10.1080/10508422.2018.1467764, Ethics \& Behavior, 1-18 (2018)

Greening, D.W. y B. Gray, Testing a model of organizational response to social and political issues, Academy of Management Journal, 37, 467-498 (1994)

Hair, J., R. Anderson, R. Tatham y W. Black, Análisis Multivariante, 5ª Ed., Prentice Hall, Madrid, España (1999)

Hernández, R., C. Fernández y M.P. Baptista, Metodología de la Investigación, 5aㅡ Ed., McGraw Hill Educación, México D.F., México (2010)

Johnson, M.P. y S. Schaltegger, Two decades of sustainability management tools for SMEs: How far have we come?, doi: 10.1111/jsbm.12154, Journal of Small Business Management, 54(4), 481-505 (2016)

Judge, T.A. y R.F. Piccolo, Transformational and transactional leadership: A meta-analytic test of their relative validity, doi: 10.1037/0021-9010.89.5.755, Journal of Applied Psychology, 89, 755-768 (2004) 
Larrieta, I., E. Velasco-Balmaseda, S. Fernández, M.M. Almeida y G. Intxaurburu-Clemente, Does having women managers lead to increased gender equality practices in corporate social responsibility?, doi: 10.1111/beer.12081, Business Ethics: A European Review, 24, 91-110 (2015)

Lee, J., B. Almanza y otros tres autores, Does transformational leadership style influence employees' attitudes toward food safety practices?, doi: 10.1016/j.ijhm.2012.09.004, International Journal of Hospitality Management, 33, 282-293 (2013)

Lee, J., Effects of leadership and leader-member exchange on commitment, doi: 10.1108/01437730510633728, Leadership \& Organization Development Journal, 26(8), 655-672 (2005)

Lepoutre, J. y A. Heene, Investigating the impact of firm size on small business social responsibility: A critical review, doi: 10.1007/s10551-006-9183-5, Journal of Business Ethics, 67(3), 257-273 (2006)

Luu, T., Corporate social responsibility, leadership, and brand equity in healthcare service, doi: 10.1108/17471111211247929, Social Responsibility Journal, 8(3), 347-362 (2012)

Maak, T., N. Pless y C. Voegtlin, Business Statesman or Shareholder Advocate? CEO Responsible Leadership Styles and the Micro-Foundations of Political CSR, doi: 10.1111/joms.12195, Journal of Management Studies, 3(53), 463-493 (2016)

Marcus, A.A. y M.H. Anderson, A general dynamic capability: Does it propagate business and social competencies in the retail food industry?, doi: 10.1111/j.1467-6486.2006.00581.x, Journal of Management Studies, 43, 19-46 (2006)

Marquis, C., M.A. Glynn y G.F. Davis, Community isomorphism and corporate social action, doi: 10.5465/amr.2007.25275683, Academy of Management Review, 32, 925-945 (2007)

Mhatre, K.H. y R.E. Riggio, Charismatic and transformational leadership: Past, present, and future, doi: 10.1093/oxfordhb/9780199755615.013.012, The Oxford Handbook of Leadership and Organizations, $221-240$ (2014)

Mohamed, L.M., Assessing the effects of transformational leadership: A study on Egyptian hotel employees, Journal of Hospitality and Tourism Management, 27, 49-59 (2016)

O'Neill, J.W. y K. Davis, Work stress and well-being in the hotel industry, doi: 10.1016/j.ijhm.2010.07.007, International Journal of Hospitality Management, 30, 385-390 (2011)

Padilla, C.P., D.A. Arévalo, M.A. Bustamante y C.L. Vidal, Responsabilidad Social Empresarial y Desempeño Financiero en la Industria del Plástico en Ecuador, doi: 10.4067/S0718-07642017000400012, Revista Información Tecnológica, 28(4), 93-102 (2017)

Serenko, A. y N. Bontis, A citation-based ranking of the business ethics scholarly journals, doi: 10.1504/IJBGE.2009.023790, International Journal of Business Governance and Ethics, 4, 390-399 (2009)

Stevens, J.M., H.K. Steensma, D.A. Harrison y P.L. Cochran, Symbolic or substantive document? The influence of ethics codes on financial executives' decisions, doi: 10.1002/smj.440, Strategic Management Journal, 26, 181-195 (2005)

Sully de Luque, M.S., N.T. Washburn, D.A. Waldman y R.J. House, Unrequited profit: How stakeholder and economic values relate to subordinates' perceptions of leadership and firm performance, doi: 10.2189/asqu.53.4.626, Administrative Science Quarterly, 53, 626-654 (2008)

Waddock, S.A. y C. Bodwell, Total Responsibility Management: The Manual, Greenleaf Publishing, Sheffield, Inglaterra (2007)

Waldman, D.A. y D. Siegel, Defining the socially responsible leader, doi: 10.1016/j.leaqua.2007.12.008, Leadership Quarterly, 19, 117-131 (2008)

Waldman, D.A., M.S. de Luque, N. Washburn y R.J. House, Cultural and leadership predictors of corporate social responsibility values of top management: a globe study of 15 countries, doi: 10.1057/palgrave.jibs.8400230, Journal of International Business Studies, 37(6), 823-837 (2006)

Weaver, G.R., L.K. Treviño y P.L. Cochran, Corporate ethics programs as control systems: Influences of executive commitment and environmental factors, doi: 10.5465/256873, Academy of Management Journal, $42,41-57$ (1999)

Wood, D.J., Measuring corporate social performance: a review, doi: 10.1111/j.1468-2370.2009.00274.x, International Journal of Management Reviews, 12(1), 50- 84 (2010) 
